\title{
Boundary conditions for the influence of spatial proximity on context-specific attentional settings
}

\author{
Nathaniel T. Diede ${ }^{1}$ (D) Julie M. Bugg ${ }^{1}$ \\ Published online: 19 February 2019 \\ (C) The Psychonomic Society, Inc. 2019
}

\begin{abstract}
Flexibility of cognitive control is illustrated by the context-specific proportion compatibility (CSPC) effect, the now welldocumented pattern showing that compatibility effects are reduced in mostly incompatible relative to mostly compatible locations. The episodic-retrieval account attributes the CSPC effect to location-specific representations that include the attentional settings formed via experience within a given location (e.g., a "focused" attentional setting becomes bound to a location with frequent conflict, whereas a "relaxed" setting becomes bound to one with infrequent conflict). However, Diede and Bugg (Attention, Perception, \& Psychophysics, 78, 1255-1266, 2016) demonstrated that the attentional setting associated with a given location can be based on experiences that accumulate across multiple "grouped" locationsnamely, those that are proximal to each other, relative to other (distal) locations. This spatial grouping effect supported the relative-proximity hypothesis, which we further tested in the present study. Experiment 1 replicated the spatial grouping effect and showed that it could be disrupted by a horizontal line dividing the otherwise grouped locations. Experiments 2 through 4 suggested that grouping might be a form of "chunking"- that is, the spatial grouping effect did not occur when the proximal locations were few enough in number (two) to represent independently, but it did occur when there were six locations. When there were eight proximal locations (and ten locations overall), the CSPC effect disappeared entirely. These findings suggest important boundary conditions for the relative-proximity hypothesis and inform our understanding of how past experiences with conflict are organized in the form of episodic representations that enable on-the-fly adjustments in cognitive control.
\end{abstract}

Keywords Context-specific cognitive control $\cdot$ Proportion congruence $\cdot$ Flanker $\cdot$ Spatial proximity

In the past decade or so, it has been demonstrated that attention is controlled not just strategically or willfully, as had long been assumed, but also by one's environment, motivating the oxymoronic notion of "automatic" control (Jacoby, Lindsay, \& Hessels, 2003), as well as "reactive" (Braver, Gray, \& Burgess, 2007) and "on-the-fly" control (Lehle \& Hübner, 2008). These notions challenge the traditional controlled/

Electronic supplementary material The online version of this article (https://doi.org/10.3758/s13414-019-01686-8) contains supplementary material, which is available to authorized users.

Julie M. Bugg

jbugg@wustl.edu

Nathaniel T. Diede

ndiede@wustl.edu

1 Department of Psychological and Brain Sciences, Washington University, St. Louis, MO, USA automatic processing dichotomy (Bugg \& Crump, 2012; cf. Awh, Belopolsky, \& Theeuwes, 2012) and raise the question: How could attention be controlled in this fashion? The general idea is that our past experiences with stimuli shape our future interactions with those stimuli, and in some cases with related stimuli. More specifically, the attentional settings we use when interacting with stimuli time and time again become bound to those stimuli, along with other characteristics of the episode, such as the context in which the stimuli occurred. Encountering those stimuli or contexts in the future can trigger retrieval of the associated settings, thereby reactively controlling attention.

The general question we sought to explore in the present study is how the internal representations of our prior experiences with stimuli are stored and organized in order to enable reactive control (cf. Abrahamse, Braem, Notebaert, \& Verguts, 2016, for current theorizing about the role of learning and memory processes in cognitive control) and, in particular, context-driven modulations of control. We 
investigated this question using the flanker task in the context of a modified context-specific proportion compatibility ${ }^{1}$ (CSPC) paradigm, which we will describe momentarily. In the standard CSPC paradigm, participants encounter flanker stimuli that are randomly presented across two locations (i.e., contexts; Crump, Gong, \& Milliken, 2006). One location (e.g., lower on the screen) is mostly incompatible, such that most trials comprise stimuli for which the central target arrow points in a different direction from its flanking arrows. The other location (e.g., higher on the screen) is mostly compatible, such that most trials comprise stimuli for which the central arrow matches the flanking arrows. The compatibility effect (i.e., the detriment to performance on incompatible as compared to compatible trials) is smaller in the mostly incompatible location than in the mostly compatible location, and this pattern is termed the CSPC effect (cf. Eriksen \& Eriksen, 1974). This effect has been interpreted as demonstrating a reactive, context-driven modulation of attention based on the history of conflict in a location (Crump \& Milliken, 2009).

Several findings have converged to suggest that the CSPC effect represents a genuine modulation of attention and that the control mechanism operates reactively. That it is genuine is supported by findings demonstrating that it cannot be attributed to the priming of responses following an incompatible stimulus (Crump et al., 2006; cf. Mayr, Awh, \& Laurey, 2003) or to a pure stimulus-response learning mechanism (Diede \& Bugg, 2017). That it is reactive is based on various features of the paradigm: The overall proportion of compatible to incompatible stimuli is $50 \%$, and there is a $50 \%$ likelihood that a given stimulus will appear in either context (lower vs. upper), such that participants cannot anticipate prior to stimulus onset which attentional setting should be engaged (see Bugg \& Crump, 2012). Instead, it is assumed that the presentation of a stimulus rapidly triggers retrieval of the appropriate setting (cf. Shedden, Milliken, Watter, \& Monteiro, 2013). Indeed, if a uniform attentional setting were applied throughout the task (e.g., proactively), a CSPC effect would not be observed - the compatibility effect would be equivalent across locations. Although there is some evidence that the attentional setting adopted in the mostly incompatible location is associated with more effort than the setting adopted in the mostly compatible location (Diede \& Bugg, 2017), context-specific modulations of control appear to occur implicitly. Participants have been unable to retrospectively report differences in the proportions of compatible trials between contexts in CSPC paradigms (Crump, Vaquero, \& Milliken, 2008; Diede \& Bugg, 2016, 2017; but see Schouppe, Ridderinkhof, Verguts, \& Notebaert, 2014).

The unique feature of the modified CSPC paradigm used in the present study is that multiple locations appear in the lower and upper halves of the screen, instead of just one location per

\footnotetext{
${ }^{1}$ Also known as the context-specific proportion congruence paradigm (Crump, Gong, \& Milliken, 2006).
}

half. All locations within a given half share the same history of conflict (e.g., all three locations in the lower half are mostly incompatible; see Diede \& Bugg, 2016). The advantage of the modified paradigm is that it enables one to examine how experiences across multiple locations, including those in the opposite half of the screen (i.e., those with the opposite proportion compatibility), may contribute to learning of the attentional setting for an individual location. The significance of this question is evident when considering the episodicretrieval account and extant models of cognitive control.

According to the episodic-retrieval account, the CSPC effect occurs because contextual cues (e.g., locations) become bound with the attentional settings repeatedly used in each context, in the form of episodic representations (Crump \& Milliken, 2009; see also Bugg \& Crump, 2012; Crump, 2016). When a contextual cue is later encountered, these representations are automatically retrieved, and the associated attentional setting is applied. The mostly incompatible location cues a control setting that filters the flanking arrows to a greater degree than the control setting cued by the mostly compatible location. The episodic-retrieval account can explain the speed and flexibility of the CSPC effect, as well as transfer of attentional settings to novel stimuli that appear within a "trained" location (Crump, Brosowsky, \& Milliken, 2017; Crump \& Milliken, 2009; though see Hutcheon \& Spieler, 2017).

A key assumption of the episodic-retrieval account and extant models of cognitive control that accommodate CSPC effects (Verguts \& Notebaert, 2008; cf. Blais, Robidoux, Risko, \& Besner, 2007) is that the history of conflict (i.e., proportion compatibility) is a key determinant of attentional control settings. That is, if asked to predict what setting should be retrieved when a stimulus is presented in location $\mathrm{X}$, the models would consult the history of conflict within that location (i.e., the accumulation of conflict signals for a given context). The models do not currently consider the possibility that conflict signals from nearby locations might also contribute to the learning of attentional settings (i.e., to episodic representations). However, this is important, because the attentional setting that is retrieved for an individual location may or may not coincide with the proportion compatibility of that location, depending on how representations of experiences with conflict across locations are stored and organized. Consider the following case - a mostly incompatible location on the bottom half of the screen is adjacent to a mostly compatible location on the top half of the screen. The locations are just below and above the (invisible) midline (i.e., fixation), respectively. The models predict a CSPC effect, such that the compatibility effect is smaller for the mostly incompatible location. However, it has recently been demonstrated that this prediction may not always be accurate.

The primary evidence countering this prediction stems from a "spatial grouping effect" that was found within the modified CSPC paradigm (Diede \& Bugg, 2016, Exp. 1). 
When two mostly compatible and two mostly incompatible locations were placed in opposite halves of the screen but were more proximal to one another relative to distal locations that shared their respective proportion compatibilities, the proximal (middle) locations were spatially grouped (see the left side of Fig. 1a for an illustration; cf. Corballis \& Gratton, 2003, where relative proximity was held constant). In other words, although the distal, outer locations elicited a CSPC effect, the proximal, middle locations did not. (Note that the term spatial grouping effect is used hereafter to refer specifically to the "grouping" of the middle locations and the corresponding absence of a CSPC effect for these locations.)

The relative-proximity hypothesis was proposed to explain the spatial grouping effect (Diede \& Bugg, 2016). The hypothesis extended the episodic-retrieval account by suggesting that locations of greater relative proximity, despite their differing proportion compatibilities (conflict histories), are bound into a single episodic representation separate from the distal locations. Because the middle locations contributing to the episodic representation in Diede and Bugg (2016) had opposite conflict histories (e.g., 25\% compatible vs. $75 \%$ compatible locations), the summed experience of all trials within the group (i.e., across the four locations) resulted in a 50\% proportion compatibility. The resulting attentional setting associated with and retrieved by any of the locations falling within the group was therefore an "unbiased" setting (i.e., intermediate to the settings one would find in a mostly incongruent and a mostly incongruent location), thereby precluding the CSPC effect. In support of the relative-proximity hypothesis, when the middle locations were separated so that they were most proximal to locations with the same conflict history (i.e., the distal [outer] locations in each half), the spatial grouping effect disappeared (Diede \& Bugg, 2016, Exp. 2; see Fig. 1b). These findings highlight the need for a greater understanding of how conflict experiences within and across locations are stored and organized, thereby influencing the content of episodic representations, and consequently attentional control.

\section{Present study}

In the present study, we sought to identify the conditions under which a given location is bound with an attentional setting that maps directly onto the history of conflict for that singular location, versus a setting that incorporates experiences with conflict across multiple locations (i.e., a group). This aim was achieved by conducting five experiments further testing the relative-proximity hypothesis. Across the experiments, we examined how the presence of different types of cues in terms of relative proximity affect the organization and representation of conflict experiences, and therefore the tendency to (or not to) spatially group the middle locations. To preview our findings, we replicated and extended the spatial grouping effect to novel conditions not previously tested, in addition to identifying boundary conditions for the relative-proximity hypothesis.

A secondary aim of the present experiments was to measure explicit awareness of the differences in proportion compatibility between the lower and upper halves of the screen, since prior studies had demonstrated that CSPC effects may occur in the absence of explicit awareness of proportion compatibility. In all experiments, the participants were asked to estimate the proportion of compatible trials in the lower and upper halves of the screen separately, as well as to provide a confidence rating for each estimate (Crump et al., 2008; Diede \& Bugg, 2016, 2017). Analyses of these data are reported in the main text of the Results sections. Participants were also asked to choose which half of the screen "felt more difficult." The results of these "choice" analyses were consistent with the other data, and for brevity, they are available in the supplementary material.

\section{Experiment 1}

In Experiment 1 we adopted the modified CSPC design of Diede and Bugg (2016), shown on the left side of Fig. 2a, in

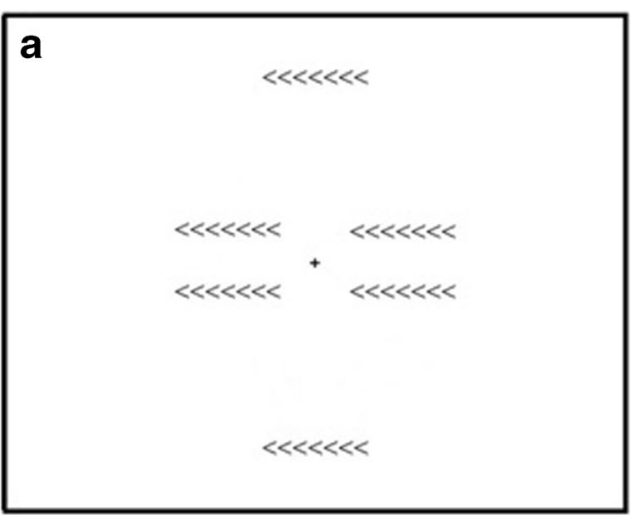

Fig. 1 Two arrangements from Diede and Bugg (2016): (a) one in which the spatial grouping effect was found for the middle locations, and (b) one in which the spatial grouping effect was not found. Only one stimulus was

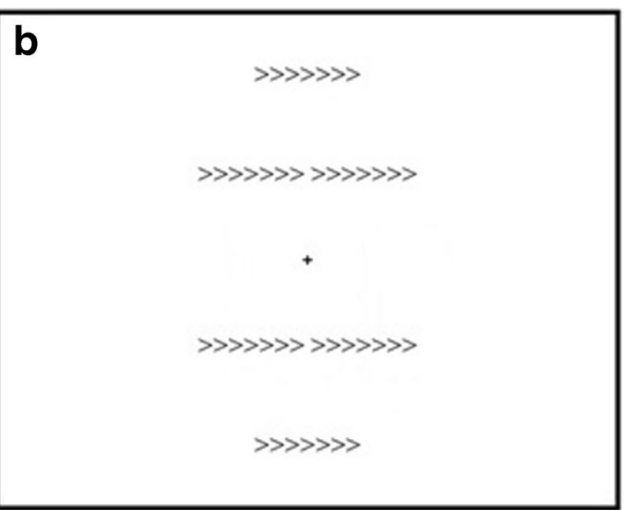

presented per trial, and all locations appearing below fixation had one proportion compatibility (PC; e.g., mostly incompatible) and all appearing above fixation had the opposite PC (e.g., mostly compatible) 
a
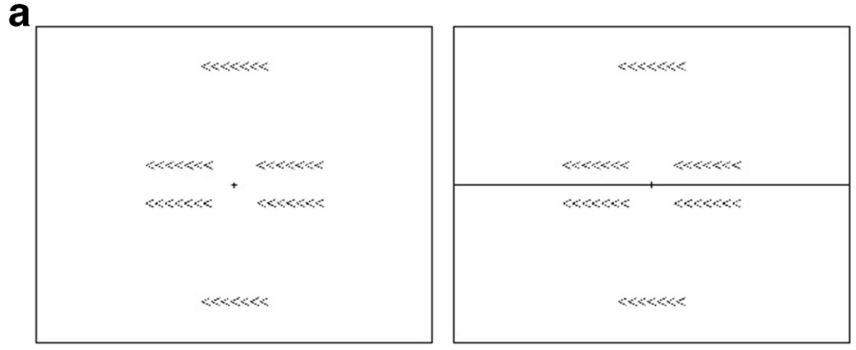

C
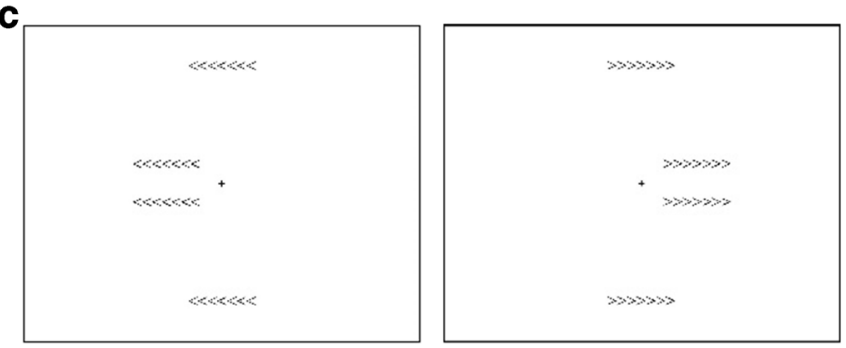

Fig. 2 Arrangements of locations in (a) Experiment 1, (b) Experiment 2, (c) the two counterbalance conditions of Experiment 3, and (d) Experiments $4 \mathrm{a}$ (left) and $4 \mathrm{~b}$ (right). Only one stimulus was presented

which the spatial grouping effect was observed, supporting the relative-proximity hypothesis. The primary manipulation in Experiment 1 was the presence or absence of a horizontal line on the computer screen. We predicted that presence of this line would cue participants to create distinct episodic representations for the middle locations appearing below fixation and the middle locations appearing above fixation. If this prediction were confirmed, the lower and upper middle locations should trigger the retrieval of unique attentional settings - a mostly incompatible (focused) attentional setting for the locations below fixation, and a mostly compatible (relaxed) control setting for the locations above fixation. Consequently, the spatial grouping effect should not be observed (i.e., there should be a CSPC effect for the middle locations). If this pattern were observed, it would suggest that the effect of relative proximity can be "overridden" by other, perhaps more salient features of the environment (e.g., a perceptual border that may function as a cue for organizing space and the corresponding episodic representations of conflict), therefore highlighting an important boundary condition within which the relative-proximity hypothesis may hold influence. In the line-absent condition, we expected that we would observe the spatial grouping effect (as evidenced by the absence of a CSPC effect for middle locations), replicating Diede and Bugg (2016).

\section{Method}

Participants A sample of 62 participants was recruited. The participants were required to be right-handed, have normal or corrected-to-normal vision, and be between 18 and 25 years of age. The data from two participants were excluded: One participant did not follow instructions and responded incorrectly

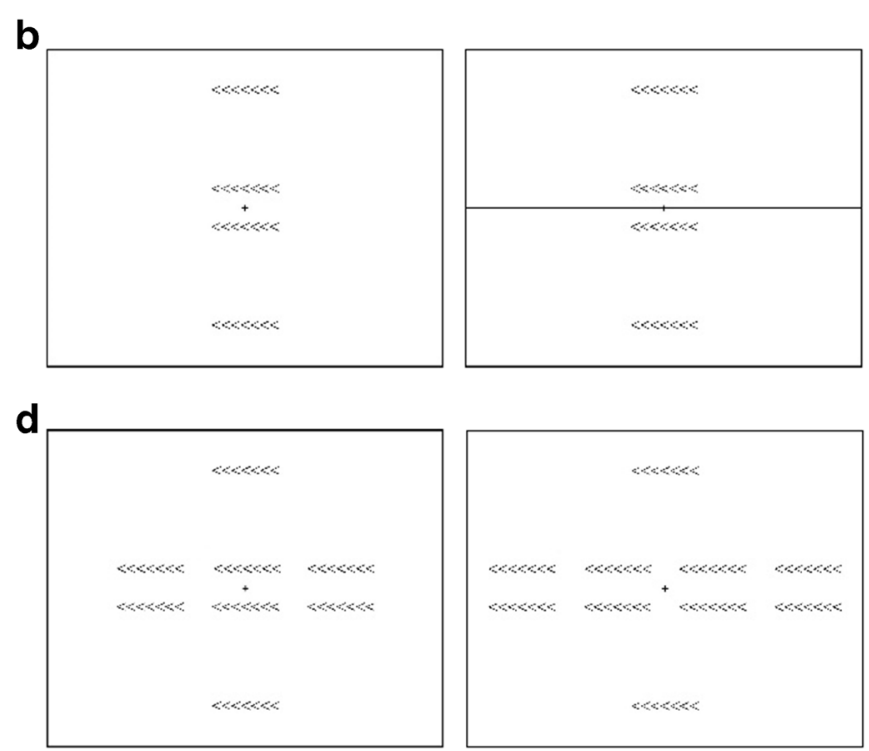

per trial. All locations appearing below fixation had one proportion compatibility (PC; e.g., mostly incompatible), and all appearing above fixation had the opposite PC (e.g., mostly compatible)

on every incompatible trial, whereas the other had an average reaction time greater than three $S D$ s above the mean. Partial course credit was given as compensation for our participants' time.

Stimuli Flanker stimuli were composed of seven arrows that could point either up, down, left, or right. The flanker stimuli were $5.6 \mathrm{deg}$ wide by $0.7 \mathrm{deg}$ tall. A central fixation cross was $0.5 \mathrm{deg}$ wide by $0.5 \mathrm{deg}$ tall. The two distal outer locations were located $9.1 \mathrm{deg}$ above and below the central fixation cross. The middle locations were located $4.5 \mathrm{deg}$ from the central fixation cross, $8.6 \mathrm{deg}$ from all other stimuli in a given half of the screen, and $2.9 \mathrm{deg}$ from the nearest location in the opposite half (see Fig. 2a; Diede \& Bugg, 2016). In the linepresent condition, a horizontal line $0.08 \mathrm{deg}$ in width bisected the screen through the fixation cross.

Design A $2 \times 2 \times 2$ mixed design was used, with trial type (compatible vs. incompatible) and proportion compatible (mostly compatible vs. mostly incompatible) as withinsubjects variables and line presence (present vs. absent) as a between-subjects variable. There were four possible compatible stimulus arrangements and 12 possible incompatible arrangements (e.g., an up-facing central arrow surrounded by down-, right-, or left-facing arrows). All 16 stimulus arrangements were presented in all locations. Only one stimulus was presented per trial. The proportion of compatible trials was manipulated by half of the screen (counterbalanced across participants). In the mostly compatible half, all locations had a $75 \%$ probability of presenting a compatible stimulus across trials, whereas the other $25 \%$ of trials would be incompatible. The probabilities were reversed in the mostly incompatible 
half. Half of the participants had a horizontal line on the screen during the experimental trials, and the other half did not.

Procedure Participants were tested in a small room, seated approximately $70 \mathrm{~cm}$ from a $1,280 \times 1,024 \mathrm{LCD}$ monitor. A research assistant was seated to the right of participants, initially gaining consent and administering a demographics questionnaire. Participants were then instructed by the computer to respond as quickly and accurately as possible to the central arrow of the flanker stimulus. Their responses were made on the keyboard number pad, where the " 8 ," " 4 ," "6," and " 2 " keys corresponded to "up," "left," "right," and "down" responses. The keys were covered with white labels. The research assistant demonstrated how to respond using the keys, emphasizing that participants should use only their right index finger and return that finger to the central " 5 " key between responses. Twelve randomly presented practice trials were presented centrally, followed by five experimental blocks. A break was allowed between blocks.

Following Diede and Bugg (2016), the experimental blocks consisted of 96 randomly presented trials. Within each block, each half of the screen presented a total of 48 trials, with each location presenting 16 trials. A stimulus was presented until response, after which a fixation cross was presented for 1,000 ms. For those in the line-present condition, the horizontal line was always on screen - during stimulus presentation and during fixation. Reaction times and error rates were recorded. Following the task, the research assistant administered a debriefing questionnaire. The questionnaire asked participants to estimate the proportions of compatible to incompatible trials in the "top portion" and the "bottom portion" of the screen separately, and to make sure that each estimate totaled to $100 \%$. After each estimate, participants rated their confidence in the estimate on a 9-point Likert scale, on which 1 equaled not at all confident and 9 equaled completely confident. Next they were asked whether they felt that "one portion of the screen was more difficult than the other," given the options "both portions were equally difficult," "the top portion was more difficult," and "the bottom portion was more difficult." Finally, participants were asked the same question again, but now they were not given the option to choose "both portions were equally difficult," forcing them to choose either the top or the bottom portion. The research assistant then debriefed the participants, with the whole procedure lasting approximately $30 \mathrm{~min}$.

\section{Results}

For this and all subsequent experiments, trials on which errors were committed and trials with reaction times (RTs) less than $200 \mathrm{~ms}$ or greater than $2,000 \mathrm{~ms}$ were excluded from the analysis (Diede \& Bugg, 2016). Trimming of the RTs eliminated $0.5 \%$ of all trials in Experiment 1 . The overall rate of committing an error was low $(M=1.7 \%, S D=1.4 \%)$. The analysis of error rates did not contradict the RT patterns and is reported in the supplementary material. An alpha level of .05 was used for all inferences. Partial eta-squared $\left(\eta_{\mathrm{p}}{ }^{2}\right)$ and generalized eta squared $\left(\eta_{\mathrm{G}}{ }^{2}\right)$ were used as the effect sizes (Lakens, 2013; Olejnik \& Algina, 2003). In addition, Bayes factors were calculated for theoretically important null effects (using JASP version 0.8.3.1), to assess the amount of evidence in favor of including a factor against the prior probability of including that factor (see Wagenmakers et al., 2018, for more information about this analysis). The $r$ scale for fixed effects was set to 0.5 in JASP. The Bayes factor used to interpret the results $\left(B F_{\text {inclusion}}\right)$ represents the degree of evidence for including that factor in an explanatory model, as compared to the summed prior probability of including the factor across all possible models. A Bayes factor greater than 1 indicates stronger evidence in favor of including a factor in an explanatory model, whereas a Bayes factor less than one indicates greater evidence in favor of excluding the factor. For example, a $B F_{\text {inclusion }}$ of 2 would indicate that the given factor predicted the data two times better than did the prior. Inversely, a $B F_{\text {inclusion }}$ of 0.5 would indicate that the prior model predicted the data two times better than the given model did. A $B F_{\text {inclusion }}$ of 1 would indicate equivocal evidence for both models. Table 1 reports the mean RTs for all analyses, whereas Table 2 reports the respective error rates. Besides the effects reported below, no other effects were significant. The data from Experiment 1 and all other experiments are available on the Open Science Framework: https://osf.io/8f4em/

All locations A 2 (trial type) $\times 2$ (proportion compatible) $\times 2$ (line presence) mixed-design analysis of variance (ANOVA) was conducted across all locations in a given half of the screen. We found an overall CSPC effect, as indicated by a significant Trial Type $\times$ Proportion Compatible interaction, $F(1,58)=20.11, M S E=196, p<.001, \eta_{\mathrm{p}}{ }^{2}=.257, \eta_{\mathrm{G}}{ }^{2}=$ .004 , due to a larger compatibility effect in the mostly compatible half $(M=184 \mathrm{~ms})$ than in the mostly incompatible half ( $M=168 \mathrm{~ms})$. As is illustrated in the left panel of Fig. 3, this effect was not modulated by the presence of the horizontal line, as indicated by a nonsignificant three-way interaction and a Bayes factor favoring the null hypothesis, $F<1, \eta_{\mathrm{p}}{ }^{2}=$ $.014, \eta_{\mathrm{G}}{ }^{2}<.001, B F_{\text {inclusion }}=.025$. An overall compatibility effect was found, indicated by a significant main effect of trial type, $F(1,58)=1,238.54, M S E=1,503, p<.001, \eta_{\mathrm{p}}{ }^{2}=.955$, $\eta_{\mathrm{G}}{ }^{2}=.625$.

Outer locations Moving to the middle panel of Fig. 3, using a $2 \times 2 \times 2$ mixed-design ANOVA, the same pattern was observed when analyzing only the outer locations. A significant CSPC effect was found, $F(1,58)=10.72, M S E=756, p=$ $.002, \eta_{\mathrm{p}}{ }^{2}=.156, \eta_{\mathrm{G}}{ }^{2}=.007$, due to a larger compatibility effect in the outer mostly compatible location $(M=157 \mathrm{~ms})$ 
Table 1 Mean reaction times within each proportion compatibility and trial type

\begin{tabular}{|c|c|c|c|c|c|c|}
\hline \multirow[t]{2}{*}{ Experiment } & \multirow[t]{2}{*}{ Line presence } & \multirow[t]{2}{*}{ Locations } & \multicolumn{2}{|c|}{ Mostly compatible } & \multicolumn{2}{|c|}{ Mostly incompatible } \\
\hline & & & Compatible & Incompatible & Compatible & Incompatible \\
\hline \multirow[t]{6}{*}{1} & \multirow[t]{3}{*}{ Absent } & All locations & $617(60)$ & 797 (79) & $662(64)$ & 789 (78) \\
\hline & & Outer locations & $644(63)$ & 803 (79) & $664(74)$ & $792(79)$ \\
\hline & & Middle locations & $603(61)$ & $793(80)$ & $601(62)$ & 787 (79) \\
\hline & \multirow[t]{3}{*}{ Present } & All locations & $637(80)$ & $824(88)$ & $646(76)$ & $816(86)$ \\
\hline & & Outer locations & $670(84)$ & $825(87)$ & $679(87)$ & $819(92)$ \\
\hline & & Middle locations & $620(80)$ & $825(93)$ & $629(76)$ & $814(84)$ \\
\hline \multirow[t]{6}{*}{2} & \multirow[t]{3}{*}{ Absent } & All locations & $558(62)$ & $687(82)$ & $568(65)$ & $678(83)$ \\
\hline & & Outer locations & $614(67)$ & $776(84)$ & $626(68)$ & $763(83)$ \\
\hline & & Middle locations & $531(61)$ & $647(85)$ & $538(67)$ & $636(85)$ \\
\hline & \multirow[t]{3}{*}{ Present } & All locations & $596(103)$ & $718(107)$ & 602 (99) & $706(100)$ \\
\hline & & Outer locations & $650(109)$ & 801 (104) & $656(96)$ & $788(91)$ \\
\hline & & Middle locations & $569(101)$ & $679(112)$ & $575(105)$ & $667(104)$ \\
\hline \multirow[t]{3}{*}{3} & \multirow[t]{3}{*}{ Absent } & All locations & $586(62)$ & $754(84)$ & $596(67)$ & $740(74)$ \\
\hline & & Outer locations & $634(64)$ & $814(93)$ & 645 (67) & 797 (74) \\
\hline & & Middle locations & $562(63)$ & $724(83)$ & $572(68)$ & $711(76)$ \\
\hline \multirow[t]{3}{*}{$4 a$} & \multirow[t]{3}{*}{ Absent } & All locations & $608(69)$ & 777 (78) & 609 (67) & 768 (73) \\
\hline & & Outer locations & $640(72)$ & $801(88)$ & $646(67)$ & $784(75)$ \\
\hline & & Middle locations & 597 (69) & $770(78)$ & 597 (69) & $763(74)$ \\
\hline \multirow[t]{3}{*}{$4 b$} & \multirow[t]{3}{*}{ Absent } & All locations & $666(108)$ & 846 (116) & $672(110)$ & 847 (113) \\
\hline & & Outer locations & 685 (113) & 847 (122) & 697 (106) & 843 (113) \\
\hline & & Middle locations & $661(107)$ & 845 (118) & $666(112)$ & 848 (114) \\
\hline
\end{tabular}

Values outside of parentheses are means, those within are standard deviations

than in the outer mostly incompatible location ( $M=134 \mathrm{~ms})$. The horizontal line again did not significantly affect the CSPC effect, $F(1,58)=1.21, M S E=756, p=.276, \eta_{\mathrm{p}}{ }^{2}=.020, \eta_{\mathrm{G}}{ }^{2}=$ $.001, B F_{\text {inclusion }}=.053$. The overall compatibility effect was significant, $F(1,58)=827.67, M S E=1,534, p<.001, \eta_{\mathrm{p}}{ }^{2}=$ $.935, \eta_{\mathrm{G}}^{2}=.514$.

Middle locations The critical pattern was found when analyzing the middle locations using a $2 \times 2 \times 2$ mixed-design ANOVA, shown in the right-hand panel of Fig. 3 . Participants who were presented with a horizontal line had a 21-ms CSPC effect for the middle locations, whereas those who were not had only a 4-ms effect. This resulted in a significant three-way interaction, $F(1,58)=4.54, M S E=258, p$ $=.037, \eta_{\mathrm{p}}{ }^{2}=.073, \eta_{\mathrm{G}}{ }^{2}=.001$. The overall CSPC effect was also significant, $F(1,58)=9.63, M S E=258, p=.003, \eta_{\mathrm{p}}{ }^{2}=$ $.142, \eta_{\mathrm{G}}{ }^{2}=.002$, as was the compatibility effect, $F(1,58)=$ $1,140.20, M S E=1,927, p<.001, \eta_{\mathrm{p}}{ }^{2}=.952, \eta_{\mathrm{G}}{ }^{2}=.653$.

Estimates of proportions Participants on average estimated that there were more compatible trials in the mostly compatible half of the screen $(M=54.89 \%)$ than in the mostly incompatible half $(M=49.55 \%), F(1,57)=5.64, M S E=149, p=$
$.021, \eta_{\mathrm{p}}{ }^{2}=.090, \eta_{\mathrm{G}}{ }^{2}=.039$. However, the estimates for both halves were still significantly different from the correct proportions in the mostly compatible half (correct: $75 \%$ compatible), $t(59)=-10.16, p<.001, d=-1.31,95 \%$ CI $[-23.34,-$ 15.66], and the mostly incompatible half (correct: $25 \%$ compatible), $t(58)=15.02, p<.001, d=1.96,95 \%$ CI [21.30, $27.85] .^{2}$ The presence of a horizontal line did not have a significant effect on the overall estimates, $F(1,57)=1.77, M S E=$ $213, p=.189, \eta_{\mathrm{p}}{ }^{2}=.030, \eta_{\mathrm{G}}{ }^{2}=.018$, nor were the differences in estimates between halves significantly different, $F<1, \eta_{\mathrm{p}}{ }^{2}$ $=.030, \eta_{\mathrm{G}}{ }^{2}=.018$, for those who saw a horizontal line (mostly compatible: $M=56.57 \%$; mostly incompatible: $M=51.33 \%$ ) versus those who did not (mostly compatible: $M=53.10 \%$; mostly incompatible: $M=47.76 \%$ ). Participants' confidence in their estimates was equivalent across all analyses, all $F_{\mathbf{s}}<1$. The mean confidences for mostly compatible $(M=4.07)$ and mostly incompatible $(M=4.10)$ estimates when the horizontal line was present centered around the same value as the mostly compatible $(M=4.00)$ and mostly incompatible $(M=4.10)$ estimates when the line was absent.

\footnotetext{
${ }^{2}$ The degrees of freedom for the mostly incompatible test differ because one participant did not provide proportion estimates on this question.
} 
Table 2 Mean error rates within each proportion compatibility and trial type

\begin{tabular}{|c|c|c|c|c|c|c|}
\hline \multirow[t]{2}{*}{ Experiment } & \multirow[t]{2}{*}{ Line presence } & \multirow[t]{2}{*}{ Locations } & \multicolumn{2}{|c|}{ Mostly compatible } & \multicolumn{2}{|c|}{ Mostly incompatible } \\
\hline & & & Compatible & Incompatible & Compatible & Incompatible \\
\hline \multirow[t]{6}{*}{1} & \multirow[t]{3}{*}{ Absent } & All locations & $0.2(0.4)$ & $3.1(3.0)$ & $0.0(0.0)$ & $2.3(2.1)$ \\
\hline & & Outer locations & $0.2(0.6)$ & $1.5(2.3)$ & $0.0(0.0)$ & $1.7(2.9)$ \\
\hline & & Middle locations & $0.1(0.3)$ & $4.0(4.1)$ & $0.0(0.0)$ & $2.7(2.2)$ \\
\hline & \multirow[t]{3}{*}{ Present } & All locations & $0.4(0.8)$ & $6.8(17.2)$ & $0.2(0.6)$ & $6.4(17.4)$ \\
\hline & & Outer locations & $0.4(1.1)$ & $5.4(1.8)$ & $0.3(1.2)$ & $5.7(1.8)$ \\
\hline & & Middle locations & $0.4(0.8)$ & $7.5(17.3)$ & $0.1(0.5)$ & $6.8(17.5)$ \\
\hline \multirow[t]{6}{*}{2} & \multirow[t]{3}{*}{ Absent } & All locations & $0.1(0.3)$ & $3.2(4.0)$ & $0.3(0.7)$ & $2.4(2.7)$ \\
\hline & & Outer locations & $0.1(0.5)$ & $5.0(5.4)$ & $0.0(0.0)$ & $4.1(4.5)$ \\
\hline & & Middle locations & $0.1(0.3)$ & $2.4(3.8)$ & $0.4(1.0)$ & $1.7(2.6)$ \\
\hline & \multirow[t]{3}{*}{ Present } & All locations & $0.2(0.5)$ & $2.7(4.6)$ & $0.1(0.4)$ & $2.5(3.7)$ \\
\hline & & Outer locations & $0.1(0.5)$ & $4.9(9.4)$ & $0.0(0.0)$ & $4.6(8.3)$ \\
\hline & & Middle locations & $0.2(0.5)$ & $1.7(2.8)$ & $0.1(0.5)$ & $1.5(1.9)$ \\
\hline \multirow[t]{3}{*}{3} & \multirow[t]{3}{*}{ Absent } & All locations & $0.3(0.5)$ & $3.8(5.4)$ & $0.2(0.7)$ & $3.5(3.6)$ \\
\hline & & Outer locations & $0.2(0.7)$ & $5.0(9.0)$ & $0.2(0.9)$ & $3.4(3.6)$ \\
\hline & & Middle locations & $0.2(0.7)$ & $3.2(4.1)$ & $0.3(1.0)$ & $3.5(4.0)$ \\
\hline \multirow[t]{3}{*}{$4 a$} & \multirow[t]{3}{*}{ Absent } & All locations & $0.2(0.5)$ & $9.6(6.9)$ & $0.4(0.8)$ & $3.7(4.0)$ \\
\hline & & Outer locations & $0.0(0.0)$ & $4.1(6.6)$ & $0.0(0.0)$ & $2.7(4.6)$ \\
\hline & & Middle locations & $0.2(0.5)$ & $4.0(4.1)$ & $0.4(0.8)$ & $3.8(4.2)$ \\
\hline \multirow[t]{3}{*}{$4 b$} & \multirow[t]{3}{*}{ Absent } & All locations & $0.2(0.4)$ & $6.9(17.9)$ & $0.3(0.8)$ & $7.2(18.0)$ \\
\hline & & Outer locations & $0.0(0.0)$ & $5.0(18.6)$ & $0.3(1.5)$ & $6.2(18.3)$ \\
\hline & & Middle locations & $0.2(0.4)$ & $7.3(17.9)$ & $0.3(0.9)$ & $7.4(18.1)$ \\
\hline
\end{tabular}

Values outside of parentheses are means, those within are standard deviations

\section{Discussion}

There were two primary findings in Experiment 1. First, the results of Diede and Bugg (2016) were replicated: When no horizontal line was present, the spatial grouping effect was observed for the middle locations, as evidenced by the absence of a CSPC effect within those locations. This finding supports the relative-proximity hypothesis and reinforces the idea that episodic representations can incorporate experiences with conflict across multiple locations. The second major

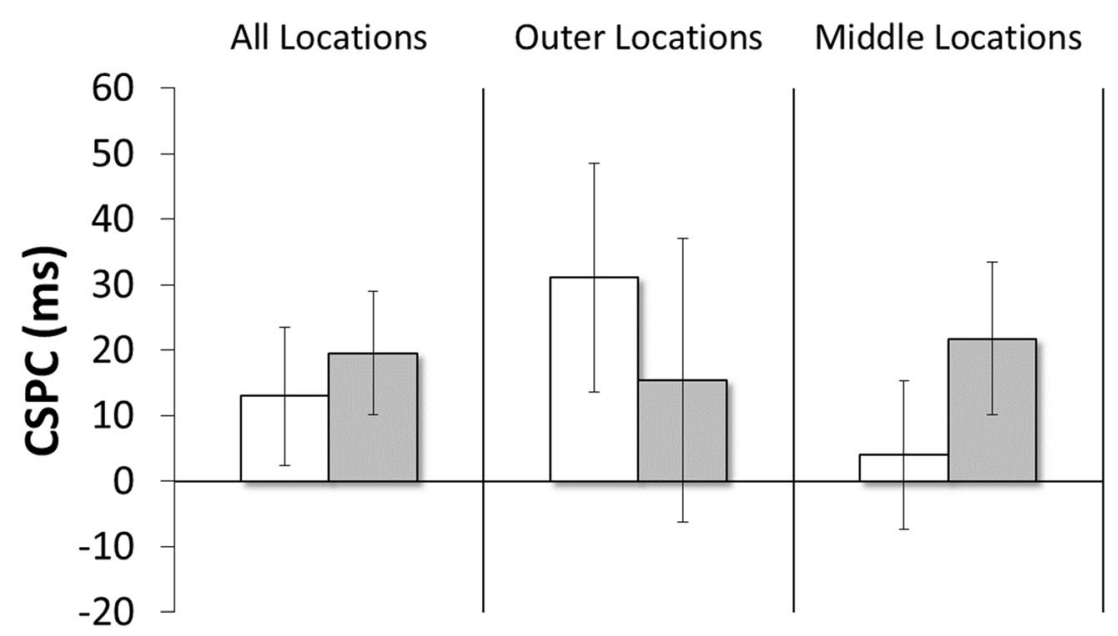

$\square$ Line Absent $\quad \square$ Line Present

Fig. 3 Context-specific proportion compatibility (CSPC) effects in the line-absent and line-present conditions of Experiment 1 . The CSPC effect was derived by subtracting the compatibility effect in the mostly incompatible context from the effect in the mostly compatible context 
finding was that the presence of a horizontal line separating the screen into lower and upper halves eliminated the spatial grouping effect, as evidenced by the presence of a CSPC effect in the middle locations. This finding extends Diede and Bugg (2016) by revealing a second method for disrupting the spatial grouping effect, in addition to spatially separating the middle locations. The horizontal line (like spatial separation in Diede \& Bugg, 2016) may have encouraged participants to organize conflict experiences according to "lower" and "upper" halves, such that the episodic representations for the middle lower and middle upper locations were bound to unique attentional settings. As in prior research (Crump et al., 2008; Diede \& Bugg, 2016, 2017), participants' estimates of the proportion of congruent trials in each half of the screen were highly inaccurate. Though the estimates for each half were in the correct direction relative to $50 \%$, low confidence ratings suggest that if awareness of CSPC existed, it was weak.

\section{Experiment 2}

Experiment 1 demonstrated one boundary condition for the relative-proximity hypothesis, by showing that an experimenter-imposed cue (e.g., a horizontal line that separated the lower and upper halves of a screen) can override the tendency for participants to spatially group proximal middle locations. Interpreted within the episodic-retrieval account, the horizontal line may have cued participants to form two episodic representations, one for the lower (bound with a mostly incompatible attentional setting) and one for the upper (bound with a mostly compatible attentional setting) half of the screen, resulting in a CSPC effect. Without the explicit cue, experiences appear to have been organized in light of the relative proximity of the middle locations, leading to three distinct episodic representations: one corresponding to the uppermost location, which was bound with a mostly compatible attentional setting; one corresponding to the middle four locations, which was bound with an unbiased (50\% compatible/ intermediate) attentional setting; and one corresponding to the lowermost location, which was bound with a mostly incompatible attentional setting. This organization explains the presence of a CSPC effect for the outermost locations but not for the middle locations in the line-absent condition.

An interesting question raised by the findings from the lineabsent condition (see also Diede \& Bugg, 2016) is why the spatial grouping effect occurs. One possible explanation is that having many locations, which have different proportions of compatibility, makes it challenging to maintain each location as a separate episodic representation. Indeed, doing so would have yielded six distinct episodic representations in Experiment 1 (see also Diede \& Bugg, 2016). In response to this challenge, the cognitive system may rely on a shortcut, namely grouping the middle locations into a single episodic representation, similar to "chunking" (Miller, 1956). An obvious means of testing this explanation would be to reduce the number of middle locations. In Experiment 2 we did just this, by collapsing the middle locations in each half such that only one middle location and one outer location was presented per half of the screen (see Fig. 2b). If the number of middle locations were reduced, then each location might be maintained distinctly, obviating the need for chunking, and thereby resulting in no spatial grouping effect (i.e., the presence of a CSPC effect). However, if relative proximity is the primary determinant of spatial grouping, then spatial grouping would persist even in the presence of fewer middle locations. As in Experiment 1, when a horizontal line separated the lower and upper halves of the screen (and consequently, the lower and upper middle locations), the spatial grouping effect should not occur (i.e., there should be a CSPC effect in the middle locations).

\section{Method}

Participants A new sample of 60 participants was recruited from the same pool as in Experiment 1, with the same exclusion criteria. Participants were compensated with course credit for their time.

Stimuli The stimuli were the same as in Experiment 1. The outer locations remained in the same locations relative to the fixation cross. The middle locations remained 2.9 deg from each other. Unlike in Experiment 1, though, the middle locations were located 1.6 deg above and below the fixation cross (see Fig. 2b).

Design A $2 \times 2 \times 2$ mixed design was used, with trial type and proportion compatible as within-subjects variables and line presence (present or absent) as a between-subjects variable.

Procedure The procedure was the same as in Experiment 1, with one change. Only two locations were presented per half of the screen: one outer and one middle. Each outer location presented 16 trials per block, whereas the middle location presented 32 trials per block. This was done in order to hold the numbers of trials per block and region constant across Experiments 1 and 2, while changing only the number of locations in the middle region.

\section{Results}

Trimming RTs eliminated $0.3 \%$ of trials. The overall error rate was low $(M=1.5 \%, S D=1.8 \%)$ and did not contradict the patterns seen in the RTs (see the supplementary material). See Tables 1 and 2 for the RTs and error rates in each condition. As in Experiment 1, a $2 \times 2 \times 2$ mixed-design ANOVA was used for all analyses. 
All locations The overall CSPC effect was significant, $F(1,58)$ $=30.52, M S E=167, p<.001, \eta_{\mathrm{p}}{ }^{2}=.345, \eta_{\mathrm{G}}{ }^{2}=.003$. As is shown in the left-hand panel of Fig. 4, the horizontal line again did not affect the CSPC effect when averaged across all locations, $F<1, \eta_{\mathrm{p}}{ }^{2}<.001, \eta_{\mathrm{G}}{ }^{2}<.001, B F_{\text {inclusion }}=.070$. The overall compatibility effect was significant, $F(1,58)=$ $1,010.39, M S E=806, p<.001, \eta_{\mathrm{p}}{ }^{2}=.946, \eta_{\mathrm{G}}{ }^{2}=.307$.

Outer locations The overall CSPC effect was significant for the outer locations, as well, $F(1,58)=9.04, M S E=799, p=$ $.004, \eta_{\mathrm{p}}{ }^{2}=.135, \eta_{\mathrm{G}}{ }^{2}=.004$, and the CSPC effect was again not affected by the horizontal line, $F<1, \eta_{\mathrm{p}}{ }^{2}=.002, \eta_{\mathrm{G}}{ }^{2}<$ $.001, B F_{\text {inclusion }}=.040$, as is shown in the middle panel of Fig. 4. The overall compatibility effect for the outer locations was significant, $F(1,58)=976.43, M S E=1,300, p<.001, \eta_{\mathrm{p}}{ }^{2}=$ $.944, \eta_{\mathrm{G}}^{2}=.409$.

Middle locations As is shown in the right-hand panel of Fig. 4, the same pattern was also found for the middle locations. There was a significant CSPC effect, $F(1,58)=20.97, M S E$ $=220, p<.001, \eta_{\mathrm{p}}{ }^{2}=.265, \eta_{\mathrm{G}}{ }^{2}=.002$, and this effect did not differ across line conditions, $F<1, \eta_{\mathrm{p}}{ }^{2}<.001, \eta_{\mathrm{G}}{ }^{2}<.001$, $B F_{\text {inclusion }}=.051$. That is, there was no spatial grouping effect in either condition (line present or absent). The overall compatibility effect was significant, $F(1,58)=668.62, M S E=$ $965.82, p<.001, \eta_{\mathrm{p}}{ }^{2}=.920, \eta_{\mathrm{G}}{ }^{2}=.249$.

Estimates of proportions Participants again estimated on average that there were more compatible trials in the mostly compatible half of the screen $(M=52.97 \%)$ than in the mostly incompatible half $(M=46.30 \%), F(1,57)=5.63, M S E=233$, $p=.021, \eta_{\mathrm{p}}{ }^{2}=.090, \eta_{\mathrm{G}}{ }^{2}=.037 .^{3}$ The estimates for both halves were again significantly different from the correct proportions in the mostly compatible half, $t(58)=-9.44, p<.001, d=-$ $1.23,95 \% \mathrm{CI}[-26.68,-17.35]$, and the mostly incompatible half, $t(59)=9.95, p<.001, d=1.28,95 \%$ CI $[17.23,25.91]$. The horizontal line did not significantly affect the overall estimates, $F<1, \eta_{\mathrm{p}}{ }^{2}=.006, \eta_{\mathrm{G}}{ }^{2}=.003$, nor was there a difference between halves, $F<1, \eta_{\mathrm{p}}{ }^{2}=.014, \eta_{\mathrm{G}}{ }^{2}=.009$ (line present: mostly compatible, $M=56.57 \%$; mostly incompatible, $M=51.33 \%$; line absent: mostly compatible, $M=$ $53.10 \%$; mostly incompatible, $M=47.76 \%$ ). Participants' confidence in their estimates was again equivalent across all analyses, all $F_{\mathrm{S}}<1$ (line present: mostly compatible, $M=$ 3.97; mostly incompatible, $M=4.10$; line absent: mostly compatible, $M=4.03$; mostly incompatible, $M=4.00$ ).

\section{Discussion}

The results of Experiment 2 identified a novel boundary condition for the spatial grouping effect: When only two (rather

\footnotetext{
${ }^{3}$ One participant did not give estimates for the mostly congruent half.
}

than four) middle locations were placed proximal to one another (i.e., when the number of middle and outer locations was the same), no spatial grouping effect occurred (i.e., a CSPC effect was found for the middle locations) in the line-absent condition. Given that there was not a spatial grouping effect without a line, it is unsurprising that adding a line intended to break up grouping had no effect on the CSPC.

A strict version of the relative-proximity hypothesis cannot account for the absence of a spatial grouping effect in Experiment 2 because, as in Experiment 1 and Diede and Bugg (2016), the middle locations were indeed more proximal to one another than to the distal location in each half. The findings thus suggest that an important determinant of spatial grouping is the number of locations in the middle region. In the experiments in which the spatial grouping effect had previously been observed, there were four rather than two middle locations. The number of locations might affect the ease of maintaining each location as an independent episodic representation, and thereby the likelihood of using grouping as a chunking strategy to organize conflict experiences across multiple locations. This idea was explored further in Experiments $4 \mathrm{a}$ and $4 \mathrm{~b} .^{4}$

However, first we examined an alternative explanation for why spatial grouping did not occur in Experiment 2: namely, that all locations were presented along a vertical midline. This may have inadvertently encouraged participants to organize conflict experiences across locations, including those in the middle, into "lower" and "upper" episodic representations. When four middle locations had been used in prior experiments (as in Exp. 1 and Diede \& Bugg, 2016), a "left-right" dimension was also present, possibly muddling the development of pure lower and upper representations, and consequently of the CSPC effect in the middle locations. This possibility was tested in Experiment 3.

\section{Experiment 3}

Experiment 3 was designed to examine whether the vertical midline arrangement of locations was responsible for the creation of lower and upper episodic representations, resulting in CSPC effects in all locations (including no spatial grouping effect in the middle locations) in Experiment 2. As in Experiment 2, only two middle

\footnotetext{
${ }^{4}$ The results also rule out the possibility that a relatively greater number of trials in middle than in outer locations could have been a determinant of spatial grouping. Because the original four middle locations were collapsed to make the two middle locations in Experiment 2, there was a 75\% probability of a trial appearing in the middle locations. It could be argued that the middle region was treated as a unique representation because the outer locations were rare "exceptions," encouraging the middle locations to be grouped. Given that a spatial grouping effect was not found, this explanation is not supported.
} 


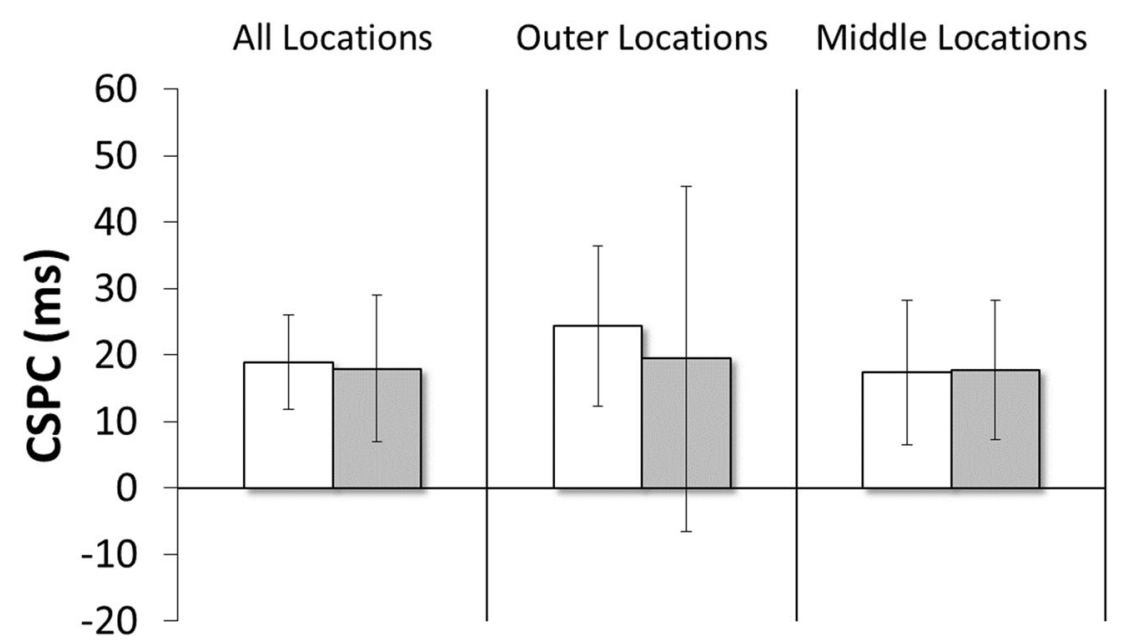

$\square$ Line Absent $\quad \square$ Line Present

Fig. 4 Context-specific proportion compatibility (CSPC) effects in the line-absent and line-present conditions in Experiment 2

locations were presented. However, the two middle locations were shifted off the vertical midline to either the left or the right (see Fig. 2c). Doing so added a lateral dimension to the locations (i.e., created a region that was offset from the vertical arrangement of the outer locations). If the vertical midline arrangement inadvertently led to distinct upper and lower representations, then disrupting that arrangement by offsetting the middle locations should lead to the reemergence of the spatial grouping effect (i.e., no CSPC effect in the middle locations). In contrast, if the number of locations is the primary factor dictating spatial grouping, the findings should replicate those of Experiment 2. Because the goal was to (re)produce the spatial grouping effect in the middle locations, the linepresent condition was dropped.

\section{Method}

Participants A new sample of 31 participants was recruited from the same pool, with the same exclusion criteria as previously. Participants were compensated with course credit for their time.

Stimuli Shown in Fig. 2c, the middle locations were offset from the fixation cross by $4.5 \mathrm{deg}$ to either the left or the right. Whether participants saw the locations to the left or right was counterbalanced. The location of these new middle locations corresponded with the left- or rightmost middle locations used in Experiment 1.

Design A $2 \times 2$ within-subjects design was used, with trial type and proportion compatible as within-subjects variables.

Procedure The procedure was the same as in Experiment 2.

\section{Results}

Tables 1 and 2 display the RTs and error rates in each condition. Trimming RTs eliminated $0.1 \%$ of trials. The overall error rate was low $(M=1.9 \%, S D=2.4 \%)$ and did not contradict the patterns seen in the RTs (see the supplementary material). A $2 \times 2$ within-subjects ANOVA was used for all analyses.

All locations A significant Trial Type $\times$ Proportion Compatibility interaction, indicating a CSPC effect, was found when averaging across all locations, $F(1,30)=21.76$, $M S E=210, p<.001, \eta_{\mathrm{p}}{ }^{2}=.420, \eta_{\mathrm{G}}{ }^{2}=.007$. The main effect of trial type - that is, the compatibility effect - was significant, $F(1,30)=668.31, M S E=1,124, p<.001, \eta_{\mathrm{p}}{ }^{2}=.957$, $\eta_{\mathrm{G}}{ }^{2}=.547$. See the left panel of Fig. 5 .

Outer locations Shown in the middle panel of Fig. 5, the outer locations also had a significant CSPC effect, $F(1,30)=7.72$, $M S E=761, p=.009, \eta_{\mathrm{p}}{ }^{2}=.205, \eta_{\mathrm{G}}{ }^{2}=.009$. The overall compatibility effect was also significant, $F(1,30)=676.51$, $M S E=1,261, p<.001, \eta_{\mathrm{p}}{ }^{2}=.958, \eta_{\mathrm{G}}{ }^{2}=.555$.

Middle locations As can be seen in the right panel of Fig. 5, there was a significant CSPC effect for the middle locations, $F(1,30)=18.67, M S E=211, p<.001, \eta_{\mathrm{p}}{ }^{2}=.384, \eta_{\mathrm{G}}{ }^{2}=.006$, indicating that the spatial grouping effect did not occur. The overall compatibility effect was significant, $F(1,30)=546.03$, $M S E=1,288, p<.001, \eta_{\mathrm{p}}{ }^{2}=.948, \eta_{\mathrm{G}}{ }^{2}=.526$.

Estimates of proportions Although participants nominally estimated more compatible trials in the mostly compatible half of the screen $(M=53.97 \%)$ than in the mostly incompatible half $(M=49.35 \%)$, the difference was not significant, $t(30)=$ $0.99, p=.331, d=0.18,95 \%$ CI $[-4.91,14.14]$. The estimates 


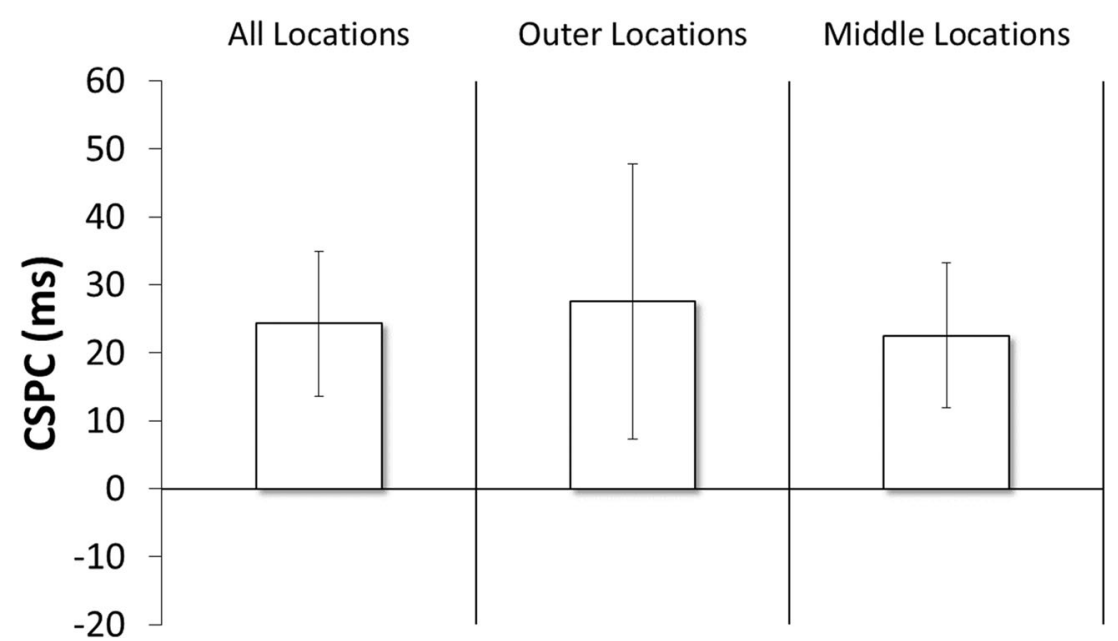

Fig. 5 Context-specific proportion compatibility (CSPC) effects across locations in Experiment 3

for both halves were again significantly different from the correct proportions in the mostly compatible half, $t(30)=-$ $6.35, p<.001, d=-1.14,95 \% \mathrm{CI}[-27.80,-14.27]$, and the mostly incompatible half, $t(30)=9.02, p<.001, d=1.62,95 \%$ CI [18.84, 29.87]. Participants' confidence in their estimates did not differ between the mostly compatible $(M=4.74)$ and mostly incompatible $(M=4.48)$ halves, $t(30)=1.31, p=.199$, $d=0.24,95 \%$ CI $[-0.143,0.660]$.

\section{Discussion}

Despite offsetting the middle locations from the vertical midline and having the middle locations occupy a distinct region of the screen, we still did not find the spatial grouping effect. The lack of spatial grouping is consistent with Experiment 2, and therefore replicates the finding that spatial grouping of middle locations is not found when only two middle locations are used. ${ }^{5}$ It is worth noting that the estimates of proportions continued to be inaccurate, even as confidence increased slightly (though it was still low overall) relative to the previous experiments.

Collectively, the data from Experiments 2 and 3 suggest that spatial grouping does not occur when only two locations are more proximal to one another than to two distal locations, a finding that cannot be accounted for by the relative-proximity hypothesis proposed by Diede and Bugg (2016). The number of proximal locations, thus, represents a major boundary condition for the effects of relative proximity on the organization of conflict experiences, and thereby on the resulting episodic representations. At present, it appears that the spatial grouping effect is

\footnotetext{
${ }^{5}$ This pattern persisted in another experiment, when we attempted to see whether reducing the number of trials in the middle locations would lead to their grouping. The procedure was the same as in Experiment 3, except that the middle locations presented only eight trials per block, whereas the outer locations continued to present 16 trials per block. The results replicated those of Experiment 3, including the lack of grouping in the middle locations (i.e., a significant CSPC effect).
}

dependent on the presence of more than two proximal locations and on the absence of a salient, experimenter-imposed cue (e.g., a horizontal line, as in Exp. 1, or increased distance between the lower middle and upper middle locations, as in Diede \& Bugg, 2016) that otherwise encourages an alternative organization of conflict experiences (i.e., separately for the lower middle and upper middle locations).

\section{Experiments $4 a$ and $4 b$}

Experiments 2 and 3 established a boundary condition for the relative-proximity hypothesis by demonstrating that the spatial grouping effect, the hallmark of the hypothesis, is not observed when the modified CSPC paradigm comprises only two middle locations. Experiments $4 \mathrm{a}$ and $4 \mathrm{~b}$ were conducted to examine whether spatial grouping occurs when the number of middle locations is increased. The only known way thus far to elicit spatial grouping has been by having a larger number of proximal (four) than of distal (two) locations. One possibility is that this precise arrangement (four to two) somehow facilitates spatial grouping, and that grouping does not occur in other arrangements. However, if spatial grouping is a byproduct of chunking, with chunking being used in situations in which it is difficult to maintain each of a large set of locations as unique locations (i.e., distinct episodic representations; cf. Luck \& Vogel, 1997, 2013, for evidence of chunking when the capacity of visual working memory is exceeded), then spatial grouping should occur regardless of the exact arrangement. In other words, there may not be an upper limit on the number of middle locations that can be accommodated (grouped) via a single (middle) episodic representation. If so, the spatial grouping effect should also occur when there are six (Exp. 4a) and when there are eight (Exp. 4b) middle locations (see Fig. 2d). This would again be evidenced by the lack of a CSPC effect in the middle locations. 


\section{Method}

Participants Two new samples, of 30 participants each, were recruited from the same pool, with the same exclusion criteria as in the preceding experiments. One participant was excluded from Experiment $4 \mathrm{~b}$ for failing to follow instructions by responding incorrectly on every incompatible trial. Participants were compensated with course credit for their time.

Stimuli In both experiments the outer locations remained unchanged. In Experiment 4a, three middle locations per half of the screen were used, as is shown on the left side of Fig. $2 \mathrm{~d}$. Each middle location was $7.5 \mathrm{deg}$ from the next closest location within the same half of the screen, and $2.9 \mathrm{deg}$ from the nearest location in the opposite half. The inner middle locations were $1.6 \mathrm{deg}$ above and below fixation, whereas the outer middle locations were $7.5 \mathrm{deg}$ from fixation. In Experiment $4 \mathrm{~b}$, four middle locations per half were used, as is shown on the right side of Fig. 2d. Each middle location was $7.5 \mathrm{deg}$ from the next closest location within the same half, and $2.9 \mathrm{deg}$ from the nearest location in the opposite half. The innermost middle locations were $4.3 \mathrm{deg}$ from fixation, whereas the outermost middle locations were $11 \mathrm{deg}$ from fixation.

Design A $2 \times 2$ within-subjects design was used, with trial type and proportion compatible as within-subjects variables.

Procedure Two notable changes were made to the procedure. First, each location presented 16 trials per block. Second, due to the increased number of trials per block, the number of blocks was reduced to four in Experiment 4a and to three in Experiment $4 \mathrm{~b}$. Although the procedures remained at $30 \mathrm{~min}$ to complete, the total trial count in Experiment 4a was slightly higher (512 trials) than in the preceding experiments (480 trials) and in Experiment $4 \mathrm{~b}$ (480 trials). A break was allowed between blocks.

\section{Results}

Tables 1 and 2 display the RTs and error rates in each condition. Trimming RTs eliminated $0.2 \%$ of trials in Experiment $4 \mathrm{a}$ and $0.8 \%$ of trials in Experiment $4 \mathrm{~b}$. The overall error rates were low in both Experiment $4 \mathrm{a}(M=3.5 \%, S D=2.5 \%)$ and $4 \mathrm{~b}(M=2.1 \%, S D=1.8 \%)$, and in neither case did they contradict the patterns seen in the RTs (see the supplementary material).

All locations In Experiment 4a (see Fig. 6), a significant CSPC effect was found when averaging across all locations, $F(1,29)$ $=5.05, M S E=156, p=.032, \eta_{\mathrm{p}}{ }^{2}=.148, \eta_{\mathrm{G}}{ }^{2}=.001$. The overall compatibility effect was significant, $F(1,29)=897.93$, $M S E=903, p<.001, \eta_{\mathrm{p}}{ }^{2}=.969, \eta_{\mathrm{G}}{ }^{2}=.575$. In Experiment $4 \mathrm{~b}$ (see Fig. 7), the CSPC effect was not significant, $F<1, \eta_{\mathrm{p}}{ }^{2}=$ $.028, \eta_{\mathrm{G}}{ }^{2}<.001, B F_{\text {inclusion }}=.223$, but the overall compatibility effect was still significant, $F(1,28)=758.38, M S E=$ $1,211, p<.001, \eta_{\mathrm{p}}^{2}=.964, \eta_{\mathrm{G}}^{2}=.397$.

Outer locations In Experiment 4a, the CSPC effect for the outer locations was marginally significant, $F(1,29)=3.49$, $M S E=1,161, p=.072, \eta_{\mathrm{p}}{ }^{2}=.107, \eta_{\mathrm{G}}{ }^{2}=.006, B F_{\text {inclusion }}=$ .907 , though the 23 -ms effect was comparable with those in the preceding experiments. The overall compatibility effect, $F(1,29)=396.53, M S E=1,684, p<.001, \eta_{\mathrm{p}}{ }^{2}=.932, \eta_{\mathrm{G}}{ }^{2}=$ .499 , was significant. In Experiment 4b, the CSPC effect for the outer locations did not reach significance, $F(1,28)=2.13$, $M S E=875, p=.155, \eta_{\mathrm{p}}{ }^{2}=.071, \eta_{\mathrm{G}}{ }^{2}=.001, B F_{\text {inclusion }}=.323$, but the overall compatibility effect, $F(1,28)=260.25, M S E=$ $2,641, p<.001, \eta_{\mathrm{p}}^{2}=.903, \eta_{\mathrm{G}}^{2}=.322$, was significant.

Middle locations Most critically, spatial grouping was found for the middle locations in Experiment 4a, as indicated by a nonsignificant CSPC effect of $7 \mathrm{~ms}, F(1,39)=1.74, M S E=$ $161, p=.198, \eta_{\mathrm{p}}{ }^{2}=.057, \eta_{\mathrm{G}}^{2}<.001, B F_{\text {inclusion }}=.259$. The overall compatibility effect was significant, $F(1,29)=773.85$,

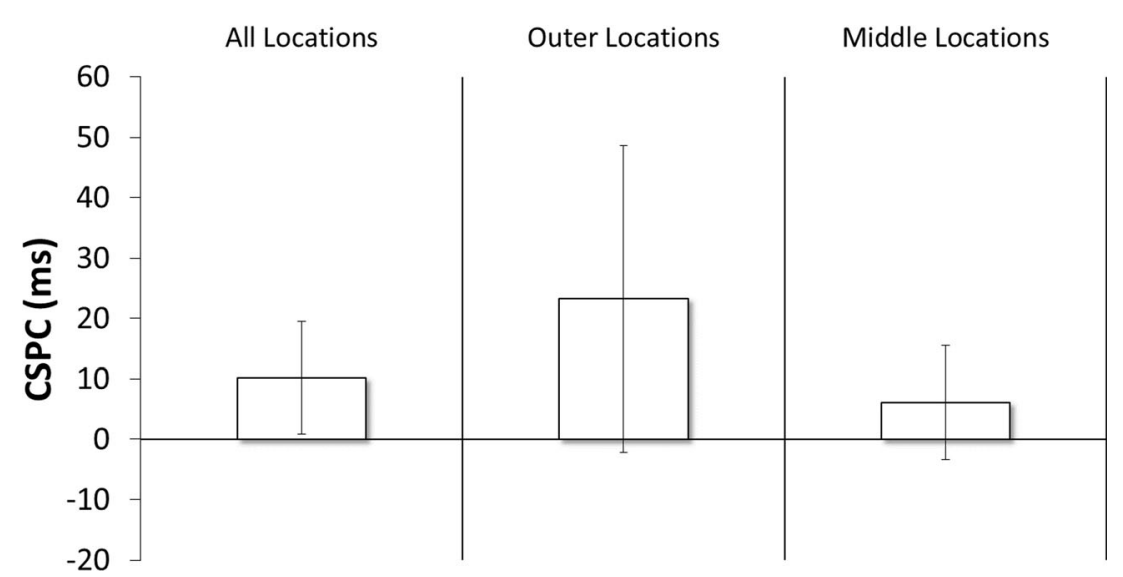

Fig. 6 Context-specific proportion compatibility (CSPC) effects across locations in Experiment 4a 


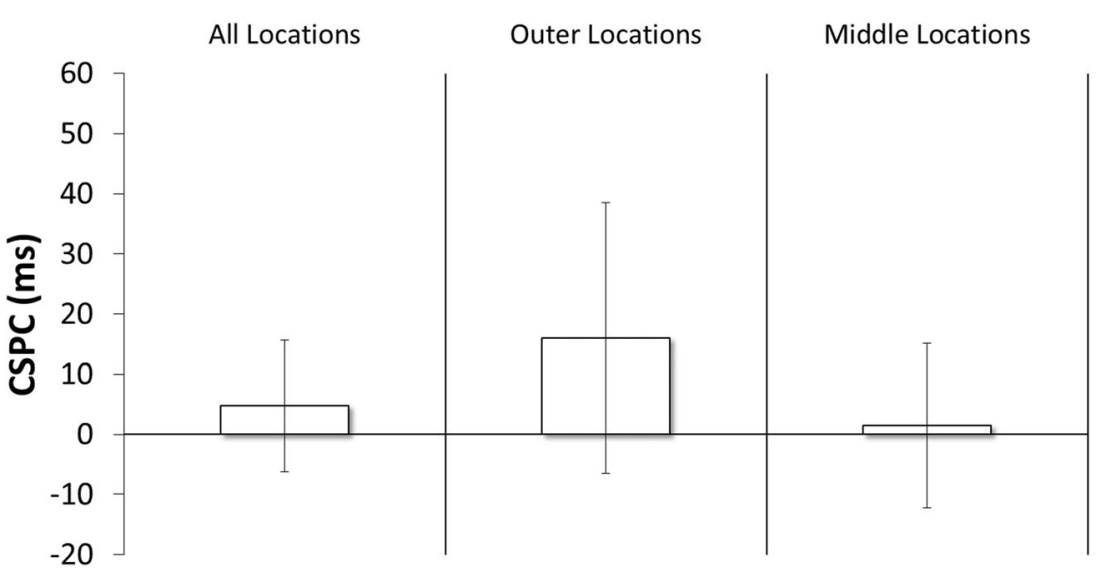

Fig. 7 Context-specific proportion compatibility (CSPC) effects across locations in Experiment 4b

$M S E=1,115, p<.001, \eta_{\mathrm{p}}{ }^{2}=.964, \eta_{\mathrm{G}}{ }^{2}=.586$. The same was true in Experiment 4b: The CSPC effect was not significant $(M=2 \mathrm{~ms}), F<1, \eta_{\mathrm{p}}{ }^{2}=.002, \eta_{\mathrm{G}}{ }^{2}<.001, B F_{\text {inclusion }}=.220$, whereas the overall compatibility effect was significant, $F(1$, $28)=797.53, M S E=1,226, p<.001, \eta_{\mathrm{p}}{ }^{2}=.966, \eta_{\mathrm{G}}{ }^{2}=.408$.

Estimates of proportions In Experiment 4a, the difference in estimates between the mostly compatible half of the screen $(M$ $=51.17 \%)$ and the mostly incompatible half $(M=48.17 \%)$ was not significant, $t(29)=0.82, p=.420, d=0.15,95 \%$ CI [$4.49,10.49]$. Estimates for both halves significantly differed from the correct proportions in the mostly compatible half, $t(29)=-7.94, p<.001, d=-1.45,95 \%$ CI $[-29.97,-$ 17.70], and the mostly incompatible half, $t(29)=8.01, p<$ $.001, d=1.46,95 \%$ CI $[17.25,29.08]$. Participants' confidence in their estimates did not differ between the mostly compatible $(M=3.30)$ and mostly incompatible $(M=2.97)$ halves, $t(30)=0.33, p=.169, d=0.25,95 \%$ CI $[-0.150$, 0.817]. In Experiment 4b, the difference in estimates between the mostly compatible half of the screen $(M=54.72 \%)$ and the mostly incompatible half $(M=51.03 \%)$ was not significant, $t(28)=1.03, p=.312, d=0.19,95 \%$ CI $[-3.65,11.03]$. Estimates for both halves significantly differed from the correct proportions in the mostly compatible half, $t(28)=-6.73$, $p<.001, d=-1.25,95 \%$ CI $[-26.44,-14.11]$, and the mostly incompatible half, $t(28)=7.41, p<.001, d=1.38$, $95 \%$ CI $[18.84,33.23]$. Participants' confidence in their estimates did not differ between the mostly compatible $(M=3.66)$ and mostly incompatible $(M=3.79)$ halves, $t(28)=-0.55, p=$ $.588, d=0.10,95 \%$ CI $[-0.654,0.378]$.

\section{Discussion}

The spatial grouping effect (i.e., the absence of a CSPC effect) was found for the middle locations when the number of those locations was six (Exp. 4a) and eight (Exp. 4b). Although at first blush this appears to suggest that (a) participants tend to group conflict experiences across all middle locations when there are at least four middle locations and (b) there may be no upper limit on the number of middle locations that can be grouped, several observations point to a potentially more complex interpretation. In Experiment 4a, there was a CSPC effect when collapsing across all locations, but the CSPC effect for the outer locations, though nominally comparable to those in the preceding experiments, was only marginally significant. More striking was the fact that in Experiment $4 b$, there was not a significant CSPC effect, either for the outer locations or overall. These findings raise the possibility that there was simply a general weakening of the CSPC effect in these experiments rather than a spatial grouping effect in the middle locations (i.e., an absence of the CSPC effect selectively in the middle locations), especially in Experiment 4b. We will consider this possibility further in the General Discussion.

\section{Cross-experimental exploratory analysis ${ }^{6}$}

Hutcheon, Spieler, and Eldar (2017, Exp. 1) reported an analysis of sequential congruency effects (SCE) within the CSPC paradigm. The SCE refers to a reduction in the compatibility effect following a previous trial that is incompatible as compared to compatible. Using the standard CSPC paradigm, in which there is a single location in each half of the screen, Hutcheon et al. found an SCE for "location repeat" trials (e.g., the previous and current trials appear in the same [upper] location) but not for "location switch" trials (e.g., the previous trial appears in the lower location and the current trial in the upper one), suggesting that unique, context-specific attentional settings were operating in each half. Generalizing the logic behind their analysis to the present study using the modified CSPC paradigm, we examined whether a single, unique control setting was operating for upper and middle locations within the same half. If there were a single control setting, then an SCE should be found not only for location repeat

\footnotetext{
${ }^{6}$ We thank Thomas Hutcheon for suggesting this analysis.
} 
trials, which are necessarily in one half of the screen, but also for location switch trials, which are defined here as those appearing in different locations within the same half of the screen. On the basis of the standard analyses reported throughout this article, one might expect this prediction to be confirmed in all experiments except Experiment 1, in which the presence of the horizontal line may have modulated the effect. Specifically, when the line was absent (i.e., when the spatial grouping effect was evidenced), an SCE might not be found for the location switch trials, because moving from the upper to the middle locations in the same half might constitute a change from one location-specific attentional setting (e.g., upper mostly congruent) to another (e.g., middle unbiased/intermediate). Another potential exception is Experiment $4 \mathrm{a}$, in which the spatial grouping effect was also observed, although the marginal CSPC in the outer locations muddied interpretation of that pattern.

To provide the most powerful test of the initial, broader prediction, we collapsed the data from all experiments except Experiment $4 \mathrm{~b}$ (because no CSPC was found there). We coded each trial as a repeat or switch between locations within a given half (we considered outer to outer or middle to middle to be location repeats, and middle to outer or outer to middle to be location switches). Then we performed a 2 (location switch: repeat or switch) $\times 2$ (proportion compatibility) $\times 2$ (previous compatibility) $\times 2$ (current compatibility) $\times 4$ (experiment) ANOVA on current-trial RTs. Here we selectively report the effects that correspond to the empirically derived predictions above, based on Hutcheon et al. (2017; see Table 3 for the means). We observed a significant SCE (i.e., Previous Compatibility $\times$ Current Compatibility interaction), $F(1,173)$ $=30.83, M S E=1,150, p<.001, \eta_{\mathrm{p}}{ }^{2}=.151, \eta_{\mathrm{G}}{ }^{2}=.151$, such that the compatibility effect was smaller following an incompatible than following a compatible trial. Consistent with the prediction motivated by Hutcheon et al., the SCE did not interact with location switch, $F(1,173)=0.55, M S E=$ $1,011, p=.461, \eta_{\mathrm{p}}{ }^{2}=.003, \eta_{\mathrm{G}}{ }^{2}=.003, B F_{\text {inclusion }}=.022 .^{7}$ In other words, the SCEs were similar for location repeat and location switch trials within a given half. These effects (Previous Compatibility $\times$ Current Compatibility interaction and Previous Compatibility $\times$ Current Compatibility $\times$ Location Switch interaction) did not interact with the experiment factor $\left(F \mathrm{~S}<1, B F_{\text {inclusion }} \leq .001\right){ }^{8}$

\footnotetext{
${ }^{7}$ At first glance, a reader might interpret these findings as inconsistent with Hutcheon et al. (2017), who found that there was not an SCE in the location switch condition but there was an SCE in the location repeat condition. However, in Hutcheon et al.'s study, a location switch was always necessarily a context switch (because there was only one location within each half of the screen). In the present analysis, a location switch and a location repeat both were trials in which the context was the same - that is, a change from a location within one half to a location within the same half of the screen.

${ }^{8}$ Following Hutcheon et al. (2017), we did not analyze error rates because errors were rare (only $2.5 \%$ of trials).
}

Next, to test the more nuanced prediction regarding Experiment 1, we analyzed the data from Experiment 1, using the same ANOVA without the factor experiment, but including the additional factor line presence (absent or present). Again, we found a significant SCE, $F(1,58)=7.13, M S E=$ $1,231, p=.010, \eta_{\mathrm{p}}^{2}=.109, \eta_{\mathrm{G}}^{2}=.105$, such that the compatibility effect was smaller following an incongruent than following a congruent trial, and the SCE did not interact with location switch, $F(1,58)=0.66, M S E=1,380, p=.419, \eta_{\mathrm{p}}{ }^{2}=$ $.011, \eta_{\mathrm{G}}{ }^{2}=.011, B F_{\text {inclusion }}=.008$. The SCE was not modulated by line presence, $F(1,58)=2.53, M S E=1,231, p=.117$, $\eta_{\mathrm{p}}^{2}=.042, \eta_{\mathrm{G}}{ }^{2}=.037, B F_{\text {inclusion }}=.014$, nor did the SCE interact with location switch and line presence, $F(1,58)=$ $0.12, M S E=1,380, p=.729, \eta_{\mathrm{p}}{ }^{2}=.002, \eta_{\mathrm{G}}{ }^{2}=.002$, $B F_{\text {inclusion }}=.000$.

To summarize, the results of the cross-experiment analysis indicated that there was an SCE, that it was equivalent for location repeat and location switch trials in the same half of the screen, and that this pattern was similar across Experiments 1 through $4 \mathrm{a}$. These findings suggest that a single, unique attentional setting was operating within each half of the screen. That is, the attentional setting that was used when responding to stimuli in the outer location was the same one used when responding to stimuli occurring in the middle locations within the same half. This interpretation converges with our interpretation of the findings from Experiments 1 through 4a, with two exceptions. First, for Experiment 1, we suggested that there might be a different attentional setting for the outer and middle locations within each half. In particular, we suggested that this might have been the case in the line-absent condition, in which the spatial grouping effect was found. However, the SCE analysis did not reveal differences among the experiments, and the more targeted analysis of Experiment 1 that included the factor line presence did not show that the presence of the line modulated the SCE effect across location repeat and location switch conditions. On the one hand, this is inconsistent with the idea that distinct attentional settings (i.e., one for the upper and one for the middle) operate within a given half in the line-absent condition. However, we interpret the absence of this effect with caution, because the test might have been underpowered (only Exp. 1 showed that the spatial grouping effect varied depending on the presence of the line, and thus we had a relatively small sample size for testing what might have been an interaction with a small effect size), and the means were consistent with the patterns one would expect if three settings were operating in the line-absent condition. In particular, whereas the SCE (the average decrease in the compatibility effect for trials in which the previous trial was incompatible as opposed to compatible) averaged at least $10 \mathrm{~ms}$ in all conditions in all experiments, the one exception was the location switch condition in the lineabsent condition in Experiment 1 ( $\mathrm{SCE}=-1 \mathrm{~ms}$ ).

Regarding Experiment 4a, in which the spatial grouping effect was again observed, the mean SCEs are less suggestive 
of an alternative explanation. That is, the SCEs were comparably large in the location switch and location repeat conditions. Applying the logic of Hutcheon et al. (2017), this challenges our interpretation that there might have been three attentional settings operating in Experiment $4 \mathrm{a}$. We will discuss these findings further in the General Discussion.

\section{General discussion}

We set out to test the relative-proximity hypothesis and the conditions under which the spatial grouping effect is observed in the modified CSPC paradigm. In addition to replicating the original spatial grouping effect in Experiment 1, the present findings highlighted two clear and one tentative boundary condition for the hypothesis. First, the spatial grouping of the middle locations based on their relative proximity did not occur when an experimenter-imposed cue (a horizontal line) was present and physically separated the proximal (lower middle and upper middle) locations. This is consistent with the findings of Diede and Bugg (2016), who showed that proximity could be disrupted by increasing the distance (space) between the middle locations (i.e., moving the lower and upper middle locations closer to the outer locations in each half). What is compelling about the present finding is that the middle locations were more proximal to one another, but the horizontal line still disrupted the effect of relative proximity. Interpreted within the episodic-retrieval account, the horizontal line may have encouraged participants to organize conflict experiences according to "lower" and "upper" halves, such that the episodic representations for the middle lower and middle upper locations were bound to unique attentional settings, therefore resulting in a CSPC effect.

The second boundary condition for the relative-proximity hypothesis is suggested by the findings of Experiments 2 and 3: namely, that the spatial grouping effect did not occur when there were only two proximal (one lower middle and one upper middle) locations. This was true whether the middle locations were vertically oriented (in line with the outer locations) or offset (i.e., the two locations appeared to the left of fixation or to the right of fixation). The simplest explanation is that the two middle locations were not grouped because there were only two such locations. In other words, to the extent that spatial grouping is analogous to chunking, the benefit of grouping may be that it enables the system to bypass an otherwise apparent limitation (i.e., number of locations for which one can maintain distinct episodic representations). When only two locations exist, there may be no need for spatial grouping. In contrast, when four locations exist (as in Diede \& Bugg, 2016, and the replication in Exp. 1), maintaining four episodic representations that bind proportion compatibility to the associated attentional setting may exceed memory's capacity. 
The idea of "location management," or grouping as a type of chunking, was further put to the test in Experiments $4 \mathrm{a}$ and 4b. According to this idea, when the number of middle locations is increased relative to Experiment 1, location management should again be challenged, and the spatial grouping effect should be observed. The data were partially consistent with this idea. When the number of middle locations increased to six, we found a CSPC effect overall, a marginally significant CSPC effect in the outer locations, and a spatial grouping effect in the middle locations (Exp. 4a). However, when the number increased to eight, the CSPC effect was no longer detectable in any comparison (Exp. 4b). This can be interpreted in one of two ways. It may mean that the tendency is to form one large group accommodating all locations when there are "too many" locations. In this case, because the proportion compatibility was $50 \%$ averaged across all locations (i.e., all ten locations that appeared throughout the experiment), an unbiased attentional setting was presumably retrieved for each location in this large group, resulting in no CSPC effect overall, in the outer locations, or in the middle locations. Indeed, the ten locations in Experiment $4 \mathrm{~b}$ represented the maximal number of locations per experiment in the present study and elsewhere in the CSPC literature, with one exception to our knowledge. Weidler and Bugg (2016) used 12 locations (they also included an additional eight transfer locations in the final block, which were not part of the present study). However, the locations were organized for participants according to an experimenter-imposed bulls-eye ring that was always present on screen (i.e., four locations appeared inside each of three rings with each ring representing a different proportion compatibility). Thus, it is unsurprising that a CSPC effect was found in their study but not in Experiment $4 \mathrm{~b}$, which lacked external, environmental support for organizing conflict experiences across the ten locations.

Another possible interpretation of Experiment $4 \mathrm{~b}$ is that one large group was formed not because there were too many locations, but because participants did not gain enough experience to organize the ten locations according to their proportion compatibility or the proportion compatibility of nearby locations. According to this interpretation, with enough experience, participants would eventually form and maintain distinct episodic representations for the outer locations (resulting in a CSPC effect) and ultimately group the eight middle locations (resulting in the spatial grouping effect). This remains to be tested in future research.

The results of Experiments $4 \mathrm{a}$ and $4 \mathrm{~b}$ tentatively suggest that there might be a third boundary condition for the relativeproximity hypothesis. When the number of middle locations increases $(>8)$, such that there are ten (or more) total locations, a CSPC effect occurs neither overall nor in the outer locations; accordingly, one cannot claim that there is a spatial grouping effect when a CSPC effect does not occur in the middle locations. Only once a CSPC effect is found for the outer locations in a display that includes at least eight middle locations can there be a stronger test of the prediction that there should not be an upper limit on the number of middle locations that can be grouped. However, as we noted earlier, there does appear to be a lower limit (as implied by Exps. 2 and 3) that influences whether participants group the middle locations.

\section{Implications for theory and models}

The relative-proximity hypothesis, as originally put forth by Diede and Bugg (2016), supported an expansion of the episodic-retrieval account to accommodate cases in which an attentional control setting does not map directly onto the history of conflict for a singular location but instead incorporates experiences with conflict across multiple locations (i.e., within a group). The spatial grouping effect (i.e., the lack of a CSPC effect for middle locations) that is observed under select conditions, as further elucidated in the present study, serves as the primary support for the relative-proximity hypothesis. The importance of identifying conditions under which this effect occurs, as well as boundary conditions, relates more broadly to the theoretical aim of understanding how the internal representations of our prior experiences with conflict (i.e., engaging attention) are organized and stored in order to enable context-specific control. This has implications for models of control (e.g., Verguts \& Notebaert, 2008; cf. Blais et al., 2007), which currently do not consider the possibility that conflict signals from nearby locations might contribute to the learning of attentional settings (i.e., formation of episodic representations for a given location), and therefore might not always accurately predict which attentional setting will be retrieved when a stimulus is encountered within a given context (location). To improve prediction accuracy, extant models could be modified so that model dynamics/parameters are constrained by the present findings (and those of Diede \& Bugg, 2016), which indicate when an individual location's history of conflict is or is not predictive of the attentional setting for that location. For the outer locations, the individual location's history of conflict was consistently predictive, except in Experiment 4b, in which we found no CSPC effect. This consistent result is unsurprising, given that there was not another location nearby to the outer locations. However, the importance of the present findings with respect to extant theory and models is especially apparent when considering the middle locations, for which nearby locations included those with the opposite proportion compatibility. For the middle locations, the individual location's history of conflict was not consistently predictive of the attentional setting associated with that location. Instead, in some cases (i.e., generally, when there were at least four middle locations and no perceptual cue to delineate the lower middle and upper middle locations), the best predictor of the attentional setting associated with a 
middle location was the average proportion compatibility of all middle locations.

What do these findings suggest about how conflict experiences are organized and stored in the form of episodic representations? At the simplest level, participants do appear to pick up on cues and use them to guide the organization of prior experiences with conflict. In addition to relative proximity, such cues include the horizontal line in one condition of Experiment 1. When the horizontal line appeared on screen and divided the lower middle and upper middle locations, it appears that participants organized conflict experiences occurring below the line into one episodic representation (e.g., mostly incongruent/focused setting) and those above the line (e.g., mostly congruent/relaxed setting) into a second episodic representation. This was true even though the locations were just as close to each other and as far from the outer locations as in the line-absent condition, in which relative proximity "directed" the organization of conflict experiences (resulting in the spatial grouping effect).

The findings are also consistent with the possibility that participants organize conflict experiences according to different categories of space (see also Weidler \& Bugg, 2016; cf. Bugg, Jacoby, \& Chanani, 2011; Bugg \& Dey, 2018; Cañadas, Rodríguez-Bailón, Milliken, \& Lupiáñez, 2013, for other categorical cues that guide attentional control). For example, in the line-present condition, participants may have organized experiences into a lower category and an upper category of space. In contrast, in the line-absent condition, experiences may have been organized into three categories: lower, middle, and upper, with each corresponding to an episodic representation (mostly incongruent/focused setting; 50\% congruent/ unbiased setting; and mostly congruent/relaxed setting, respectively). This same category-based organization might account for why a spatial grouping effect occurred when there were at least four middle locations, except in Experiment $4 \mathrm{~b}$. In Experiment 4b, according to a categorically oriented way of thinking about how conflict experiences are organized, there appeared to be only one category of space (the entire computer screen). Thinking about the results of Experiments 2 and 3 from this perspective, it may not be that participants are disinclined to "chunk" two locations for purposes of a workaround on capacity limitations. Rather, the input from just two nearby locations might not be sufficient to give the impression that those locations represent a unique category of space.

One challenge to the above interpretations stems from our exploratory analyses examining the SCE between locations within the same half of the screen (e.g., between the upper and middle locations; cf. Hutcheon et al., 2017). These analyses indicated that a single, unique attentional setting was operating within one half of the screen (i.e., for both the upper and middle locations) for all experiments included in the analysis (Exps. 1-4a). This contrasts with our interpretation that two distinct attentional settings were present within one half of the screen in the experiments (Exp. 1 line-absent condition, and Exp. 4a) in which the spatial grouping effect was found (e.g., a mostly congruent/relaxed setting in the upper location and an unbiased/intermediate in the middle location). Although we believe that this analysis approach has value in informing this issue, additional research will be needed to confirm the present findings. As we already noted in the exploratory analysis, the mean SCEs in Experiment 1 were nominally consistent with our interpretation. This was not true for Experiment 4a, though. Especially important will be future studies that are designed to examine SCEs using variants of the modified CSPC design presented herein and that include larger sample sizes and/or larger numbers of trials than the present study, so there will be sufficient power to detect potentially subtle interactions. One such interaction was already mentioned above (the interaction with line presence), but equally important are potential interactions with block. As we detailed throughout the discussion, it is assumed that participants learn attentional settings via experiences with conflict in each location. Therefore, it is possible that the differentiation of attentional settings within one half of the screen (i.e., unique settings formed for upper vs. middle locations) happens only after many trials of experience in each location. Future studies with additional blocks (more trials) can test this possibility directly, by examining interactions with block or halves of the experiment.

\section{Relevance to gestalt theory}

Our references to space, perception, and groups might imply that the present findings have implications for gestalt theory (see, e.g., Pomerantz \& Portillo, 2011). Although the relative-proximity hypothesis is reminiscent of the gestalt principle of proximity, and the effect of the horizontal line in Experiment 1 might evoke the principle of common region, we are reluctant to draw conclusions about gestalt principles per se, for two reasons. First, gestalt phenomena typically apply to statically presented stimuli in which all characteristics can be perceived continuously-for example, an image of dots arranged with various degrees of proximity. In the present study, only one stimulus was presented at a time. If participants were aware of the proximity differences between locations, this must have been based on an internal representation of space that was formed via accumulating input trial by trial, given that participants never saw the full array of locations at once. Second, gestalt phenomena typically refer to conscious perception. Even if participants were consciously aware of the spatial relations between locations, they did not seem to be aware of the proportion differences between the locations that were modulating attention and influencing their performance. 


\section{Awareness}

Regarding awareness, across experiments the participants were poor at estimating the true proportion of congruent trials in each half of the screen. If participants were able to make accurate estimates, it could be argued that some form of conscious processing might be contributing to the CSPC effect. The present data suggest that this is unlikely, and thus concur with many previous studies (Crump et al., 2008; Diede \& Bugg, 2016, 2017). Confidence ratings were also generally low for the estimates, with the means being in the lower half of the scale. Even if some signal of awareness was present, it was not strong enough to engender much confidence in participants' estimates. It might be argued that these low estimates of confidence could be an artifact of the measurement method (estimating proportions); however, participants' subjective feelings of difficulty also led to conclusions similar to their proportion estimates (see the supplementary material).

\section{Conclusion}

To conclude, two clear and a third tentative boundary condition for the relative-proximity hypothesis were identified across five experiments. Although there were clearly cases in which multiple middle locations (with conflicting PCs) were represented as a single group rather than independently (as indicated by the spatial grouping effect), consistent with the hypothesis, we also observed cases in which this did not occur. The identification of conditions under which the spatial grouping effect does and does not occur offers new insights into how conflict experiences are organized and stored, in the form of episodic representations that enable flexible, contextspecific control. This information can be used to inform future modeling efforts whose goal will be to predict the attentional setting that is retrieved for a given location. Future research also has the exciting potential to assess how people eke out meaningful organizations of conflict experiences in the presence and absence of experimenter-imposed cues, and how the ways in which individuals organize these experiences shape their subsequent attentional control.

Author note We are grateful to Jeff Shi, Ben Talisman, and Mariah Weintraub for their assistance during data collection. N.T.D. was supported by NIA training grant T32AG000030-40.

Publisher's note Springer Nature remains neutral with regard to jurisdictional claims in published maps and institutional affiliations.

\section{References}

Abrahamse, E., Braem, S., Notebaert, W., \& Verguts, T. (2016). Grounding cognitive control in associative learning. Psychological Bulletin, 142, 693-728. https://doi.org/10.1037/bul0000047
Awh, E., Belopolsky, A. V., \& Theeuwes, J. (2012). Top-down versus bottom-up attentional control: A failed theoretical dichotomy. Trends in Cognitive Sciences, 16, 437-443. https://doi.org/10. 1016/j.tics.2012.06.010

Blais, C., Robidoux, S., Risko, E. F., \& Besner, D. (2007). Item-specific adaptation and the conflict-monitoring hypothesis: A computational model. Psychological Review, 114, 1076-1086. https://doi.org/10. 1037/0033-295X.114.4.1076

Braver, T. S., Gray, J. R., \& Burgess, G. C. (2007). Explaining the many varieties of working memory variation: Dual mechanisms of cognitive control. In A. R. A. Conway, C. Jarrold, M. J. Kane, A. Miyake, \& J. N. Towse (Eds.), Variation in working memory (pp. 76-106). New York, NY: Oxford University Press.

Bugg, J. M., \& Crump, M. J. C. (2012). In support of a distinction between voluntary and stimulus-driven control: A review of the literature on proportion congruence effects. Frontiers in Psychology, 3, 367. https://doi.org/10.3389/fpsyg.2012.00367

Bugg, J. M., \& Dey, A. (2018). When stimulus-driven control settings compete: On the dominance of categories as cues for control. Journal of Experimental Psychology: Human Perception and Performance, 44, 1905-1932. https://doi.org/10.1037/xhp0000580

Bugg, J. M., Jacoby, L. L., \& Chanani, S. (2011). Why it is too early to lose control in accounts of item-specific proportion congruency effects. Journal of Experimental Psychology: Human Perception and Performance, 37, 844-859. https://doi.org/10.1037/a0019957

Cañadas, E., Rodríguez-Bailón, R., Milliken, B., \& Lupiáñez, J. (2013). Social categories as a context for the allocation of attentional control. Journal of Experimental Psychology: General, 142, 934-943. https://doi.org/10.1037/a0029794

Corballis, P. M., \& Gratton, G. (2003). Independent control of processing strategies for different locations in the visual field. Biological Psychology, 64, 191-209. https://doi.org/10.1016/S0301-0511(03) 00109-1

Crump, M. J. C. (2016). Learning to Selectively Attend From ContextSpecificAttentional Histories: A Demonstration and Some Constraints. Canadian Journal of Experimental Psychology, 70, 59-77

Crump, M. J. C., Brosowsky, N. P., \& Milliken, B. (2017). Reproducing the location-based context-specific proportion congruent effect for frequency unbiased items: A reply to Hutcheon and Spieler (2016). Quarterly Journal of Experimental Psychology, 70, 1792-1807. https://doi.org/10.1080/17470218.2016.1206130

Crump, M. J. C., Gong, Z., \& Milliken, B. (2006). The context-specific proportion congruent Stroop effect: Location as a contextual cue. Psychonomic Bulletin \& Review, 13, 316-321. https://doi.org/10. 3758/BF03193850

Crump, M. J. C., \& Milliken, B. (2009). The flexibility of context-specific control: Evidence for context-driven generalization of item-specific control settings. Quarterly Journal of Experimental Psychology, 62, 1523-1532. https://doi.org/10.1080/17470210902752096

Crump, M. J. C., Vaquero, J. M. M., \& Milliken, B. (2008). Contextspecific learning and control: The roles of awareness, task relevance, and relative salience. Consciousness and Cognition, 17, 22-36. https://doi.org/10.1016/j.concog.2007.01.004

Diede, N. T., \& Bugg, J. M. (2016). Spatial proximity as a determinant of context-specific attentional settings. Attention, Perception, \& Psychophysics, 78, 1255-1266. https://doi.org/10.3758/s13414016-1086-7

Diede, N. T., \& Bugg, J. M. (2017). Cognitive effort is modulated outside of the explicit awareness of conflict frequency: Evidence from pupillometry. Journal of Experimental Psychology: Learning, Memory, and Cognition, 43, 824-835. https://doi.org/10.1037/ $\mathrm{x} \operatorname{lm} 0000349$

Eriksen, B. A., \& Eriksen, C. W. (1974). Effects of noise letters upon the identification of a target letter in a non-search task. Perception \& Psychophysics, 16, 143-149. https://doi.org/10.3758/BF03203267 
Hutcheon, T. G., \& Spieler, D. H. (2017). Limits on the generalizability of context-driven control. Quarterly Journal of Experimental Psychology, 70, 1292-1304. https://doi.org/10.1080/17470218. 2016.1182193

Hutcheon, T. G., Spieler, D. H., \& Eldar, M. (2017). Properties of contextdriven control revealed through the analysis of sequential congruency effects. Acta Psychologica, 178, 107-113.

Jacoby, L. L., Lindsay, D. S., \& Hessels, S. (2003). Item-specific control of automatic processes: Stroop Process dissociations. Psychonomic Bulletin \& Review, 10, 638-644. https://doi.org/10.3758/BF03196526

Lakens, D. (2013). Calculating and reporting effect sizes to facilitate cumulative science: A practical primer for t tests and ANVOAs. Frontiers in Psychology, 4, 863. https://doi.org/10.3389/fpsyg. 2013.00863

Lehle, C., \& Hübner, R. (2008). On-the-fly adaptation of selectivity in the flanker task. Psychonomic Bulletin \& Review, 15, 814-818. https:// doi.org/10.3758/PBR.15.4.814

Luck, S. J., \& Vogel, E. K. (1997). The capacity of visual working memory for features and conjunctions. Nature, 390, 279-281. https://doi. org $/ 10.1038 / 36846$

Luck, S. J., \& Vogel, E. K. (2013). Visual working memory capacity: From psychophysics and neurobiology to individual difference. Trends in Cognitive Sciences, 17, 391-400. https://doi.org/10. 1016/j.tics.2013.06.006

Mayr, U., Awh, E., \& Laurey, P. (2003). Conflict adaptation effects in the absence of executive control. Nature Neuroscience, 6, 450-452.

Miller, G. A. (1956). The magical number seven, plus or minus two: Some limits on our capacity for processing information. Psychological Review, 63, 81-97. https://doi.org/10.1037/h0043158
Olejnik, S., \& Algina, J. (2003). Generalized eta and omega squared statistics: Measures of effect size for some common research designs. Psychological Methods, 8, 434-447. https://doi.org/10.1037/ 1082-989X.8.4.434

Pomerantz, J. R., \& Portillo, M. C. (2011). Grouping and emergent features in vision: Toward a theory of basic Gestalts. Journal of Experimental Psychology: Human Perception and Performance, 37, 1331-1349. https://doi.org/10.1037/a0024330

Schouppe, N., Ridderinkhof, K. R., Verguts, T., \& Notebaert, W. (2014). Context-specific control and context selection in conflict tasks. Acta Psychologica, 146, 63-66. https://doi.org/10.1016/j.actpsy.2013.11. 010

Shedden, J. M., Milliken, B., Watter, S., \& Monteiro, S. (2013). Eventrelated potentials as brain correlates of item specific proportion congruent effects. Consciousness and Cognition, 22, 1442-1455. https://doi.org/10.1016/j.concog.2013.10.002

Verguts, T., \& Notebaert, W. (2008). Hebbian learning of cognitive control: Dealing with specific and nonspecific adaptation. Psychological Review, 115, 518-525. https://doi.org/10.1037/ 0033-295X.115.2.518

Wagenmakers, E.-J., Love, J., Marsman, M., Jamil, T., Ly, A., Verhagen, J., . . Morey, R. D. (2018). Bayesian inference for psychology. Part II: Example applications with JASP. Psychonomic Bulletin \& Review, 25, 58-76. https://doi.org/10.3758/s13423-017-1323-7

Weidler, B. J., \& Bugg, J. M. (2016). Transfer of location-specific control to untrained locations. Quarterly Journal of Experimental Psychology, 69, 2202-2217. https://doi.org/10.1080/17470218. 2015.1111396 